Elisabetta Iossa and Giancarlo Spagnolo

Contracts as Threats:

On a Rationale for Rewarding A while Hoping for $B$

J anuary 2009 


\title{
Contracts as Threats: on a Rationale For Rewarding $A$ while Hoping For $B^{*}$
}

\author{
Elisabetta Iossa ${ }^{\dagger}$ and Giancarlo Spagnolo ${ }^{\ddagger}$
}

18 December 2008

\begin{abstract}
In this paper we explore theoretically the relationship between explicit and implicit/relational contracting distinguishing between the ex-ante decision to sign an explicit contract and the ex-post decision wheter to actually apply it. We show, among other things, that the relational efficient explicit contract tends to display overcontracting on tasks or qualitative requirements (A) that are verifiable but apparently of little use for the principal. The ex-post (non)implementation of such explicit contract can then be discretionally exchanged against the provision of non contractible tasks (B) that are highly valuable for the principal. An empirical implication of the result, consistent with casual observation in procurement, is that penalties for infringements established by explicit contracts are seldom exercised, even though violations take place and are easy to monitor and verify.
\end{abstract}

\section{Introduction}

The puzzles. Since Kerr's (1974) amusing list of examples and Holmstrom and Milgrom's (1991) pioneering analysis, it is well known that explicit contracts often reward low value (if not dysfunctional) easy-to-verify tasks at the expense of more valuable but hard to monitor ones. It is probably less well known that often real world parties do not enforce explicitly contracted provisions (e.g. do not impose penalties for contractual infringements) even though, by doing so, they apparently forego substantial

\footnotetext{
*For helpful comments, we wish to thank Sumon Bhaumik, Christopher Hellwig, Paul Grout, Bruno Jullien, Patrick Rey, Stephane Saussier and seminar participants at ATOM Centre, Brunel University and at the Workshop on Public-Private Partnerships in Bristol.

${ }^{\dagger}$ Dept of Economics and Finance, Brunel University; DEI, University of Rome Tor Vergata, CMPO and CEDI.

${ }_{\ddagger}^{\ddagger}$ DEI, University of Rome Tor Vergata, CEPR and Stocholm School of Economics.
} 
gains. The puzzle is therefore twofold: Why tasks of little value are often contractually specified? Why penalties for noncompliance are often not exercised?

Examples abound. In many organizations, the employees are contractually required to arrive at work every day at a certain time and to stay until another time, under the threat of losing the job. This is of little value for the organization when physical presence does not facilitate monitoring and the task can be as efficiently performed at home (think about creative non-team tasks). And many organizations then do not enforce these contractual provisions but allow instead flexible working hours. Similarly, in the academia, professors' contracts often establish a minimum number of teaching hours and other duties that the researcher is required to undertake. However, universities do not always apply these provisions: individual teaching loads are often reduced even when this requires to pay for extra teaching staff. In public procurement, penalties for noncompliance with contractual obligations are often waived: for example, a recent inquiry found that penalties for low quality provision by contractors procuring goods and services to Italian public administrations were imposed for less that $5 \%$ of the ascertained violations. ${ }^{1}$ Analogous anecdotal evidence exists for procurement of complex services in the UK (see below).

This paper. This paper provides an 'optimistic' joint explanation for these puzzling observations by analyzing the interaction between explicit and relational contracts from a novel perspective, one emphasizing the difference between the ex ante decision to sign an explicit contract and that of actually applying it ex post.

In an important recent contribution, Bernheim and Whinston (1998) emphasize that relational contracting needs discretion to punish defections and that, because of this, strategic ambiguity or in our wording undercontracting may be efficient. Avoiding to contractually regulate some valuable and contractible tasks may be optimal since discretion may facilitate punishment and thus cooperation on valuable non-contractible tasks. We show here that, if we take into account the possibility not to apply an explicit contract, then overcontracting - i.e. contractually requiring apparently low value verifiable tasks (or actions, or levels of effort) - may become the optimal strategy to facilitate relational contracting on valuable but non-contractible ones.

Our model shows that explicit contracts on the provision of very costly and completely valueless tasks may not be enforced in equilibrium but used as effective 'threats'

\footnotetext{
${ }^{1}$ Own calculations on data from the third party inspections commissioned by the Italian Public Procurement Agency (Consip) and coordinated by their team in charge of monitoring supply contracts (Team Monitoraggio delle Forniture). In particular, in the period 2005-2008 on a total of 4095 inspections a total of 1455 contractual infringement by the contractor were ascertained, but contractual remedies/penalties where only exercised in 64 of those cases, i.e. against about $4.42 \%$ of the infringements (with no significant difference across types of public administrations or geographical location, typically important in Italy because of differences in Social Capital). See Albano et al. (2008) for a general discussion (in Italian) of the possible reasons behind such low contract enforcement rate, besides the 'benevolent' one discussed in this paper.
} 
that facilitate relational contracting on crucial but non contractible tasks. With overcontracting on tasks that are verifiable, costly to the agent but of no use for the principal, the principal can exchange discretionally the non-implementation of such explicit contract against the provision of noncontractible tasks that are costly to the agent and valuable for the principal. The principal designs the explicit contract by requiring the agent to do more than what he actually needs him to do on the verifiable task. The principal further informally specifies what the agent needs to do on the unverifiable task. The principal and the agent then agree not to enforce the explicit contract on the verifiable task as long as the agent does what the principal requires him to do on the unverifiable task. In order to reduce his own incentives to deviate from the implicit agreement and enforce the contract on the verifiable task, the principal chooses a verifiable task that is costly to the agent to undertake but brings little or no benefit to himself. The optimal relational contract thus exhibits (over)contracting on the valueless tasks.

This reasoning offers a rationale for the puzzles discussed earlier: organizations do not enforce contractual provisions on working hours for committed and collaborative employees but might apply them to punish/fire employees that exploited their acquired freedom opportunistically. Universities accommodate teaching reductions for professors who excel in research or are particularly collaborative on other activities that are hard to specify in a contract or verify for a court. In case of opportunistic behaviour however, the application of the explicit contract might be used as a sanction against the professor. ${ }^{2}$ Parties involved in a long term relationship may not levy explicitly contracted penalties for infringements that do take place if that helps them not to improve collaboration on non-contractible aspects of their relationship. In procurement of complex services in the UK "There exists anecdotal evidence that the public sector can sometimes be reluctant to levy deductions for fear of spoiling the relationship with the private sector" (HM Treasury, 2006) ${ }^{3}$

\footnotetext{
${ }^{2}$ Following only what prescribed by the explicit contract can in fact be an effective weapon (hence threat) in the hands of unions when bargaining for higher wages. These "work to rule" (also named 'Italian strike', as it was apparently invented in Italy early in the previous century, or 'white strike'), have been used for example in the US automobile industry, where collective employment agreements for rather simple tasks are thousands of pages long, and contract enforcement is very efficient. See http://libcom.org/organise/work-to-rule for a nice up-to-date explanation from direct users, where one also reads the following: "Almost every job is covered by a maze of rules, regulations, standing orders, and so on, many of them completely unworkable and generally ignored. Workers often violate orders, resort to their own techniques of doing things, and disregard lines of authority simply to meet the goals of the company. There is often a tacit understanding, even by the managers whose job it is to enforce the rules, that these shortcuts must be taken in order to meet targets on time." (Bold our).

${ }^{3}$ The use of relational contracting is also stated clearly in the policy document: "The Government believes that the relationship between the public and private sector in a PFI project must always ultimately be contractual but should be overlaid with partnership working to ensure that operations are effective. In order to encourage this approach the Government will promote the development of a partnership agreement or shared vision document that sits outside of the actual PFI contract. This
} 
An important feature of overcontracting on a costly and valueless action is that it gives the option to the principal to react immediately to a deviation by the agent, by levying a fine for noncompliance with the explicit contract. This helps to discipline one party (the agent) without increasing the temptation to defect by the other (the principal) and thus transform simultaneous transactions into sequential ones. It follows that overcontracting has distributional implications: a principal with bargaining power can implement the efficient relational contract without leaving any rent to the agent and without using discretionary bonuses.

Of course, as for other inefficient contractual devices increasing punishments, the possibility of renegotiation at low cost tends to undermine the benefit of overcontracting as mechanism device to sustain relational contracting. In fact we show that the gain from overcontracting is smaller the lower the cost of renegotiating the contract. However, in our model, renegotiation does not completely undermine the benefit of overcontracting. Even when renegotiation costs are zero, overcontracting brings the benefit of reducing incentives to defect from the implicit agreement by transforming simultaneous-move games into sequential-move games. For this reason we find that overcontracting weakly dominates relational contracting where incentives are provided only through discretionary monetary transfers.

Finally, our paper offers a new explanation as to why penalties for contract infringement are often quite low, even though effective enforcement of contracted obligations would suggest to set them rather high (Becker 1968, Abreu 1988). In our model, increasing penalty for non compliance by the agent reduces the agent's incentive to defect but it raises the principal's one. In order not to tighten the principal's incentive constraint, with overcontracting contractual penalties for infringements are optimally bounded above by the cost of the contracted action for the agent.

Our results are general, but are particularly relevant for large firms and public organizations subject to what we call 'bureaucratic control', i.e. formal accountability rules that do not allow their members to operate discretionary monetary payments that have no verifiable counterpart, like an explicit supply contract that justifies the payment. Bureaucratic control thus hinders 'standard' relational contracting. However, while checking the correspondence between contracts and payments for auditors is relatively easy, verifying whether the explicit contract has then been effectively executed, whether the promised level of quality of service has been met, and if not, whether contractual remedies have been effectively exercised, is much more difficult. Bureaucratic control thus does not hinder relational contracting sustained through overcontracting and without using discretionary transfers.

We wrote above that our is an 'optimistic' explanation for why explicit contracts are often not applied, since we focus on benevolent actors and the enforcement of 'pro-

would not be legally binding but would set out the parameters of the public sector and private sector working relationship and spell out in some detail how the contract will be managed in practice." Thus an (explicit) contract is written but another (implicit) contract is implemented. 
ductive' noncontractible tasks B. However, all our results can readily be re-interpreted from a less optimistic point of view, with the principal being a 'non-benevolent' agent of a large firm or public organization exploiting his discretion to extract B, reinterpreted as 'Bribes' or 'private Benefits', in exchange for not enforcing the explicit contract between his organization and outsiders. As nonverifiable tasks, illegal gains must be part of a self-enforcing relational contracts and discretion on the ex-post execution of explicit contracts helps to enforce legal/productive as well as illegal/corrupt informal exchanges (see e.g. Kelman, 1990 and Banfield, 1975).

Relation to the literature. These results can be seen as a contribution to the economics of 'multi-tasking and job design', sparked by the seminal work of Holmstrom and Milgrom (1991). That paper stressed, among other things, that when some tasks are easy to monitor and to contract upon, whilst others are difficult or impossible, providing high powered incentives on the easily monitored tasks may lead agents to disregard the hard to monitor ones which can be crucial to the organization. The implication is that when the hard to monitor tasks are important it is then better to limit the power of incentives on easily monitored tasks. This formal result has been often related to Kerr's (1975) classic management science piece on "the folly of rewarding $\mathrm{A}$ when aiming for B". On the contrary, our results show that when one recognizes that signing and applying explicit contracts are distinct decisions, high powered incentive contracting on almost valueless tasks or quality dimensions (A) turn out to be the best way to sustain a relationship and obtain effort on other valuable tasks (B) that are observable but not contractible.

Our work is also related to various strands of literature focussing on implicit or relational contracts.

Cooperative relationships sustained by "the shadow of the future" are a fundamental governance mechanism for most forms of economic interaction and the rich long-standing literature on implicit or relational contracts reflects their importance. Scholars like Simon (1951), Macaulay (1963), Klein and Leffler (1981) and Telser (1981) stressed early that when economic transactions are not isolated but episodes of a history of frequent exchanges, informal agreements sustained by future gains form the long-term relationships will govern crucial aspects of the exchanges. The formal theory of implicit or relational contracts has then been developed by Bull (1987) and MacLeod and Malcomson $(1989,1998)$ and it has been considerably extended more recently by Levin (2003), MacLeod (2003), and Fuchs (2007). ${ }^{4}$

On the other hand, relational contracts are often embedded in a framework of explicit contracts on verifiable dimensions and the complex interaction between explicit and relational contracts is somewhat less well understood. Pearce and Stacchetti (1998) show that with coexisting explicit and implicit contracts a risk neutral principal will

\footnotetext{
${ }^{4}$ See also Calzolari and Spagnolo (2007) for an extension to multiple agents and competitive procurement, and MacLeod (2007) for an elegant survey of this literature.
} 
adjust the discretionary bonuses of the implicit agreement to further insure the risk averse agent, smoothing his income across periods. We know from Baker et al. (1994) and Schmidt and Schnitzer (1995) that effective explicit contracting may hinder relational contracts by improving parties' fallback position when the relationships break down after defections (softening the punishment that disciplines the relational contract and making explicit and relational contracts substitutes). More closely related to our paper, Baker et al. (1994) also show that there are circumstances in which the presence of an explicit contract facilitates implicit ones, so that explicit and implicit contracts complement each other. Closely related to our paper, besides the already discussed work by Bernheim and Whinston (1998), is also the informal work of Klein (2000), and the more recent work of Baker et al. (2006). ${ }^{5}$ Klein (2000) suggests that although relational contracts are indeed crucial for the smooth functioning of organizations, as non-contractible performance dimensions are present in different measure in every economic exchange, parties care a lot for the explicit contracts that constitute the framework within which relational contracts develop. In particular, he suggests that explicit contracts are designed with the explicit aim of facilitating relational ones, by limiting temptations to defect. Baker et al. (2006) formalize this argument in a relational contracting model with explicitly contracted allocation of valuable decision rights (governance structures) crucial for adaptation. They show that the efficient allocation to implement a decision rule minimizes the maximum aggregate temptation to renege on it.

The structure of the paper. Section 2.1 presents a simple stylized model to make our points. As in most previous work on relational contracts, in our analysis we assume that agents (employees) are risk neutral. Tasks may be differently costly and are independent in an agent's cost function. Thus assigning more than one task to the agent does not affect the cost for the agent of carrying out an individual task. The principal can observe the agent's effort but performance cannot be verified. Thus, it is not possible to write an explicit contract that rewards the agent on the basis of his performance. Section 2.2 studies the benchmark of 'standard' relational contracting, allowing for both sequential and simultaneous timing. Section 3 analyzes overcontracting in large firms and organizations subject to 'bureaucratic control', i.e. to limits in discretionary payments aimed at improving agents accountability, but making it hard for them to sustain standard relational contracts with suppliers. Section 4 analyzes the general case (no bureaucratic control), showing that in our set up overcontracing is generally optimal, as it facilitate relational contracts by transforming simultaneous transactions into sequential ones and by strengthening punishments following deviations. Section 5 considers renegotiation costs and short term contracting, showing that

\footnotetext{
${ }^{5}$ Somewhat less directly related is the work of Garvey (1995), Halonen (2002), Baker et al. (2001), Bragelien (2003) and Blonski and Spagnolo (2004) analyzing how a different institution, property rights on specific assets, interact with relational contracts.
} 
the facilitating effect of overcontracting on relationships never disappears. Section 6 briefly concludes.

\section{A Very Simple Model}

To make our point in the simplest possible environment, we focus on a very stylized principal-agent game. It will become apparent however that our results generalize to more complex situations such as bilateral holdup problems.

\subsection{Basic Setup}

We consider a long term (infinite horizon), bilateral, repeated interaction between a principal and an agent. Time is discrete and both parties discount future payoffs through a common and strictly positive factor $\delta<1$. Let $c_{J}(j)$ denote the agent's increasing and convex cost function of providing a certain level $j$ of effort in task (or good, service, investment of type) $J=A, B, C \ldots$, and let $v_{J}(j)$ denote the weakly concave increasing value accruing to the principal when receiving intensity $j$ of task $J$ from the agent. Denote by $w_{J}$ the per-period surplus generated by the exchange of $J$ against a monetary transfer or price $p_{j}$ paid by the principal to the agent, $w_{J}=$ $v_{J}(j)-c_{J}(j)$, and let $W_{J}=\sum_{\tau=1}^{\infty} \delta^{\tau} w_{\tau}$. Denote by $\bar{j}>0$ the maximum level of task intensity solving $w_{J}(\bar{j})=0$. Both the principal and the agent are risk neutral. ${ }^{6}$ In the basic model, they receive zero if they choose not to trade.

Consider a verifiable task, say $A$. In the absence of special regulations to govern the exchange of a verifiable task $A$, the parties have three alternatives.

First, they can write a (long or short-term) court-enforced explicit contract specifying the principal's due payment $p_{A}$, the agent's obligation to perform intensity $a$, and fines (including any repayments, damages, etc.) $F^{P}$ and $F^{\alpha}$ that a non-performing party must pay to the other.

Second, they can informally agree on an implicit relational contract to perform $a$ and to pay an informal monetary transfer $t_{A}$.

Third, they can use a mix of the two instruments (e.g. an explicitly contracted minimum level and an implicitly agreed effective level of payment).

If, instead, a task is not verifiable, then an explicit contract cannot be enforced by courts and only an implicit contract can govern its exchange, like a self-enforcing relational contract specifying the principal's due payment, the agent's obligation to perform, and some punishment phase triggered by a party's non-performance.

\footnotetext{
${ }^{6}$ Linearity in income is a convenient semplification and, with no uncertainty in the model, the degree of risk aversion plays no role.
} 
The relationship between the principal and the agent can thus be characterized by some verifiable (and court-enforceable) ingredients, e.g. the terms of an explicit contract on a verifiable task (task intensity $a$, the corresponding per-period contracted price $p_{A}$, the fines for the agent's non-performance $F^{\alpha}$ ), and by non-verifiable ingredients, the self-enforcing part of the agreement on non-verifiable (and, possibly, some verifiable) tasks and bonuses/transfers.

We let $\omega=\left\{x^{P}, x^{\alpha}, F^{P}, F^{\alpha}\right\}$ denote a generic one-period explicit contract, where $x^{P}$, and,$x^{\alpha}$ are the (vector of) actions or payment required by contract from the principal and the agent, $F^{P}$ and $F^{\alpha}$ are the (vectors of) fines for non-performance, and $\Omega=\left\{x^{P}, x^{\alpha}, F^{P}, F^{\alpha}\right\}$ is the long term version of the contract, such that $\omega$ applies every period in the future (unless it is renegotiated/modified).

In the basic model we assume that the principal and the agent can commit to a long-term contract (or that, equivalently, renegotiation cost is very high). We relax this assumption in Section 5 where we study the effect of renegotiation.

The timing of the game between the principal and the agent can be either simultaneous or sequential. ${ }^{7}$

\section{TIMING(S)}

period 0: The principal and the agent may sign an explicit contract and/or agree on an implicit/relational contract.

period 1: An infinite repetition of (one of) the following stage game(s) takes place:

\section{Stage Game 1 (simultaneous actions)}

Step 1: The principal pays any explicitly contracted transfers.

Step 2: The principal and the agent simultaneously choose verifiable and nonverifiable actions and the implicitly contracted transfer.

Step 3: The principal observes the agent 's actions and, if the agent violated the explicit contract, chooses whether or not to impose fines $F^{\alpha}$.

Step 4: The principal and the agent may sign new short term contracts or - when feasible - renegotiate existing long term ones.

\section{Stage Game 2 (sequential, the principal moves first)}

\footnotetext{
${ }^{7}$ Simultaneous actions may not be realistic when one party only operates monetary transfer. However, our results apply to more general settings where both parties' actions may be observable with delay, like bilateral specific investment.
} 
Step 1: The principal pays any explicitly and implicitly contracted transfers.

Step 2: The agent observes the transfers and chooses verifiable and non-verifiable actions (including exercising the fine $F^{P}$ in case the principal violated the explicit contract).

Step 3: The principal observes the agent's actions and, if the agent violated the explicit contract, chooses whether or not to impose the fines $F^{\alpha}$.

Step 4: The principal and the agent may sign new short term contracts or - when feasible - renegotiate existing long term ones.

\section{Stage Game 3 (sequential, the agent moves first)}

Step 1: The agent chooses verifiable and non-verifiable actions.

Step 2: The principal pays any explicitly and implicitly contracted transfers, observes the agent's actions and, if the agent violated the explicit contract, chooses whether or not to impose the fines $F^{\alpha}$.

Step 3: The agent exercises the fine $F^{P}$ in case the principal violated the explicit contract.

Step 4: The principal and the agent may sign new short term contracts or - when feasible - renegotiate existing long term ones.

We will consider the relationship between explicit and implicit contracts in this simple model under different assumptions on the institutional framework, like explicit contracting possibilities, distribution of bargaining power, renegotiation costs, and the presence or not of what we will call Bureaucratic Control. The term "Bureaucratic Control" is used to refer to organizational rules that forbid payments that are not part of an explicit contract; these rules are present in practically all large organizations to facilitate accounting controls through book auditing.

Before ending this section, consider the first best level of task $b, b_{F B}$. This maximizes $\frac{v_{B}(b)-c_{B}(b)}{1-\delta}$ leading to

$$
v_{B}^{\prime}\left(b_{F B}\right)=c_{B}^{\prime}\left(b_{F B}\right)
$$

\subsection{Benchmark: Standard Relational Contracting}

When a task $B$ is non-verifiable, explicit contracting is not possible at time 0 and a relational contract is the only potential alternative. The principal and the agent can agree on an implicit, relational contract requiring each period the agent to undertake task $B$ at level $b$ and the principal to operate a discretionary transfer $t_{B}$, with equilibrium per-period payoffs $\left\{t_{B}-c_{B}(b), v_{B}(b)-t_{B}\right\}$. The principal uses grim strategies: if the agent deviates, the principal will withhold payment forever in the future. The agent also uses grim strategies: if the principal deviates, the agent will never exert 
effort again in the future. We refer to this type of relational contracting, based on the use of discretionary transfers, as "standard relational contracting" (ST).

The principal and the agent will accept to participate to the contract if their respective expected payoff is non-negative, which gives the following participation constraints

$$
I R^{S T}-P: V=\frac{v_{B}(b)-t_{B}}{1-\delta} \geq 0
$$

and

$$
I R^{S T}-\alpha: U=\frac{t_{B}-c_{B}(b)}{1-\delta} \geq 0
$$

A necessary and sufficient condition for ( $\left.\mathrm{IR}^{\mathrm{ST}}-\mathrm{P}\right)$ and $\left(\mathrm{IR}^{\mathrm{ST}}-\alpha\right)$ is that: $t_{B} \in\left[c_{B}(b), v_{B}(b)\right]$. Given this, whether an implicit contract can be sustained in equilibrium depends, among other things, on the timing of the stage game.

Consider first the case of simultaneous actions, i.e. Stage Game 1. With a relational contract, the principal would stick to the agreed strategy of paying informal transfer $t_{B}$ in the current period if defecting is less profitable than sticking to the equilibrium strategy. By defecting and withholding the payment, the principal saves $t_{B}$ in the current period but foregoes the surplus $\frac{\delta\left(v_{B}(b)-t_{B}\right)}{1-\delta}$ in all subsequent periods, given that, following the defection, the agent will choose not to exert effort again in the future. The relational incentive constraint of the principal $\left(\mathrm{RIC}^{\mathrm{ST}}-\mathrm{P}\right)$ is then given by

$$
R I C^{S T}-P: V=\frac{v_{B}(b)-t_{B}}{1-\delta} \geq v_{B}(b),
$$

which is necessary and sufficient condition for the principal not to defect.

Note that an implication of $\left(\mathrm{RIC}^{\mathrm{ST}}-\mathrm{P}\right)$ is that the transfer paid by the principal to the agent must not be too high, i.e. $t_{B} \leq \delta v_{B}(b)$, that is, under standard relational contracting, the principal must enjoy a rent in each period of at least

$$
V_{S m l}(b) \equiv(1-\delta) v_{B}(b) .
$$

Consider now the relational incentive constraint of the agent (RIC- $\alpha$ ) which is necessary and sufficient condition for the agent not to defect. With standard relational contract, the agent would stick to the agreed strategy of exerting effort $b$ if defecting is less profitable than sticking to equilibrium strategies. By defecting and not exerting effort, the agent saves the cost $c_{B}(b)$ of undertaking the task for the principal in the current period but he foregoes the surplus $t_{B}-c_{B}(b)$ in all subsequent periods, which amount to $\frac{\delta\left(t_{B}-c_{B}(b)\right)}{1-\delta}$, given that following the defection the principal will choose not to pay $t_{B}$ again in the future. We then have

$$
R I C^{S T}-\alpha: U=\frac{t_{B}-c_{B}(b)}{1-\delta} \geq t_{B}
$$


and we note that to induce the agent to stick to the relational contract, the agent must receive a transfer $t_{B} \geq c_{B}(b) / \delta$, that is he must enjoy a rent in each period of at least

$$
U_{S m l}(b) \equiv \frac{(1-\delta) c_{B}(b)}{\delta}
$$

The Lemma below then derives the set of sustainable relational contracts that satisfies $\left(\mathrm{IR}^{\mathrm{ST}}-\mathrm{P}\right),\left(\mathrm{IR}^{\mathrm{ST}}-\alpha\right),\left(\mathrm{RIC}^{\mathrm{ST}}-\mathrm{P}\right)$ and $\left(\mathrm{RIC}^{\mathrm{ST}}-\alpha\right)$ with simultaneous timing.

Lemma 1 (Standard Relational Contracting with Simultaneous Timing) With demand for a single non-verifiable task and no explicit contracting on other tasks, if actions are simultaneous, a relational contract to sustain positive levels of the noncontractible task is sustainable in equilibrium iff:

$$
\Phi_{S m l}^{S T}(b, \delta) \equiv \delta^{2} v_{B}(b)-c_{B}(b) \geq 0 .
$$

Proof. The set of feasible relational contracts with standard relational contracting maximizes $b$ subject to the two participation constraints, $\left(\mathrm{IR}^{\mathrm{ST}}-\mathrm{P}\right)$ and $\left(\mathrm{IR}^{\mathrm{ST}}-\alpha\right)$ and the two relational constraints, $\left(\mathrm{RIC}^{\mathrm{ST}}-\mathrm{P}\right)$ and $\left(\mathrm{RIC}^{\mathrm{ST}}-\alpha\right)$. When $\left(\mathrm{RIC}^{\mathrm{ST}}-\mathrm{P}\right)$ and $\left(\mathrm{RIC}^{\mathrm{ST}}-\alpha\right)$ are satisfied, $\left(\mathrm{IR}^{\mathrm{ST}}-\mathrm{P}\right)$ and $\left(\mathrm{IR}^{\mathrm{ST}}-\alpha\right)$ are also satisfied, thus we can ignore $\left(\mathrm{IR}^{\mathrm{ST}}-\mathrm{P}\right)$ and $\left(\mathrm{IR}^{\mathrm{ST}}-\alpha\right)$. Summing across $\left(\mathrm{RIC}^{\mathrm{ST}}-\mathrm{P}\right)$ and $\left(\mathrm{RIC}^{\mathrm{ST}}-\alpha\right)$, we obtain a necessary condition for the relational incentive constraints to hold, the global relational incentive constraint (RIC), given by

$$
R I C^{S T}: \delta v_{B}(b)-c_{B}(b)(1-\delta)-t_{B} \geq 0 .
$$

Since the LHS of RIC is decreasing in $t_{B}$, the set of sustainable $b$ minimizes $t_{B}$ subject to $\left(\mathrm{RIC}^{\mathrm{ST}}-\mathrm{P}\right)$ and $\left(\mathrm{RIC}^{\mathrm{ST}}-\alpha\right)$. This gives $t_{B}=\frac{c_{B}(b)}{\delta}$ which is the value of $t_{B}$ such that $\left(\mathrm{RIC}^{\mathrm{ST}}-\alpha\right)$ is binding. Substituting for this value in $\left(\mathrm{RIC}^{\mathrm{ST}}-\mathrm{P}\right)$, we obtain expression $(5)$.

Through the use of discretionary transfers $t_{B}$, relational contracting can be help motivating the agent when explicit contracting is not possible. For given $\delta$, any task intensity $b \leq b_{S m l}(\delta)$, where

$$
b_{S m l}^{S T}(\delta)=\frac{1}{\Phi_{S m l}^{S T}(\delta)},
$$

can be sustained under simultaneous timing through standard relational contracting. Since $\Phi_{S m l}^{S T}($.$) is increasing in \delta$, the upper bound $b_{S m l}^{S T}(\delta)$ implementable through an implicit contract is increasing in $\delta$. For $\delta$ sufficiently high, the first best intensity $b_{F B}$ is sustainable. To put it differently, the minimum level of $\delta$ that is needed to ensure sustainability of first best intensity solves

$$
\delta_{S m l}^{S T}\left(b_{F B}\right)=\frac{1}{\Phi_{S m l}^{S T}\left(b_{F B}, \delta\right)}<1 .
$$


The inequality holds since $\Phi_{S m l}^{S T}\left(b_{F B}, \delta\right)$ is always positive for $\delta=1$ and negative for $\delta=0$ and since $\Phi_{S m l}(b, \delta)$ is increasing in $\delta$.

When the timing of the exchange is sequential as in STAGE GAme 2, there are no gains from defection for the principal: as the principal moves first, deviating by not paying the transfer $t_{B}$ is not profitable as the agent will not deliver $b$. The only potentially profitable deviation is the one of the agent, which implies that the only relevant relational incentive constraint is (RIC- $\alpha$ ). The following Lemma is then obtained:

Lemma 2 (Standard Relational Contracting with Sequential Timing). With demand for a single non-verifiable task and no explicit contracting on other tasks, if actions are sequential and the principal moves first (STAGE GAME 2), a relational contract to sustain positive levels of the non-contractible task is sustainable in equilibrium iff:

$$
\Phi_{S e q}^{S T}(b \delta) \equiv \delta v_{B}(b)-c_{B}(b) \geq 0
$$

where, from (5), $\Phi_{\text {Seq }}^{S T}(b) \geq \Phi_{S m l}^{S T}(b)$.

Proof. With sequential timing following Stage GAMe 2, the set of sustainable relational contracts satisfies $\left(\mathrm{IR}^{\mathrm{ST}}-\mathrm{P}\right),\left(\mathrm{IR}^{\mathrm{ST}}-\alpha\right)$ and and $\left(\mathrm{RIC}^{\mathrm{ST}}-\alpha\right)$. However, when $\left(\mathrm{RIC}^{\mathrm{ST}}-\alpha\right)$ is satisfied, $\left(\mathrm{IR}^{\mathrm{ST}}-\alpha\right)$ is also satisfied. Thus we can ignore $\left(\mathrm{IR}^{\mathrm{ST}}-\alpha\right)$. Noting that $\left(\mathrm{RIC}^{\mathrm{ST}}-\alpha\right)$ is easier to satisfy the higher is $t_{B}$, the set of sustainable $b$ is maximized at the maximum $t_{B}$ compatible with $\left(\operatorname{IR}^{\mathrm{ST}}-\mathrm{P}\right)$, which gives $t_{B}=v_{B}(b)$. Substituting for $t_{B}=v_{B}(b)$ in $\left(\mathrm{RIC}^{\mathrm{ST}}-\alpha\right)$ we obtain expression $(7)$.

Since there is no need to incentives the principal, no rent for the principal is needed. The set of effort level sustainable in equilibrium under standard relational contracting with sequential actions is then always strictly larger than under simultaneous actions, as it happens in a repeated 'trust game' if compared to a payoff-equivalent repeated simultaneous actions Prisoner's Dilemma game. That is

$$
b_{S e q}^{S T}(\delta) \geq b_{S m l}^{S T}(\delta) \text { and } \delta_{S e q}^{S T}<\delta_{S m l}^{S T} .
$$

Remark 1 In the sequential game we have assumed that first the principal pays $t_{B}$ and then the agent chooses task intensity. As the only possible deviation is from the agent, giving all the surplus to the agent by setting $t_{B}=v_{B}(b)$, minimizes the gain from defection. If we invert the sequence of moves, as in STAGE GAME 3, the relevant constraint becomes (RIC-P). The set of sustainable actions remains the same but it is now found by setting $t_{B}=c_{B}(b)$ and thus giving all the surplus to the principal. 


\section{Bureaucratic Control: (Over)Contracting on A to Obtain B}

Suppose our principal is a manager of a large organization subject to regulatory or governance rules that do not allow to operate discretionary payments $t_{B}$ that are not part of an explicit contract, say because it would be too hard then to prevent some dishonest agents present in the population to unduly appropriate part of the transfer. In this example we will define this common practice by two assumptions very close to many real world situations. 'Bureaucratic Control' (BC):

BC1. With BC, it is forbidden for the principal to operate 'discretionary transfers', i.e. transfers that are not justified by an explicit contract detailing what was that transfer paid for, and renegotiating publicly awarded explicit contract is forbidden.

This type of control rule, used in most large hierarchical organizations to facilitate internal monitoring, excludes payments against any non-verifiable services/actions, like $t_{B}$. It only allows for monetary transfers, like $p_{B}$, prescribed by an explicit contract detailing for what kind of (contractible) good of service is exchanged against the monetary transfer.

BC2. Monitoring coincidence between payments and explicit contracts in the books is easy and cheap (e.g. through book audits) and it is always done; monitoring contract implementation, i.e. verifying whether the goods or service explicitly contracted upon have been effectively delivered at the quality levels the contract prescribed, is extremely costly/difficult and it is never done.

In what follows the presence of BC will mean that these two assumptions are both satisfied. They bring to the limit, for the sake of crispness of our example, the rather obvious observation that checking whether books are correct, i.e. that to each payment corresponds an explicit contract, is much cheaper and is done much more often in organization than checking whether the explicit contracts have then been effectively managed/applied with the due care to obtain all what was paid for at the agreed quality level.

Suppose now that the principal is subject to BC, so that it cannot operate discretionary payments that are not part of an explicit contract. With $\mathrm{BC}$ imposing $t_{B}=0$, standard relational contracts on task $B$ become unfeasible: the agent will never provide effort $b$ given that he cannot be compensated for it. To emphasize:

Lemma 3 (Bureaucratic Control). With the principal subject to $B C$ and demand for a single non-verifiable task, standard relational contracting cannot sustain any trade in equilibrium, i.e. $\Phi_{\text {Seq }}^{B C}=\Phi_{S m l}^{B C}=\varnothing$. 
However, parties under BC can circumvent the reduction of discretion by writing an explicit contract on some other verifiable task $A$, that for now we make not valuable by assumption (we show later that this is indeed optimal), and use that to motivate the agent to provide the valuable but non-contractible task $B$.

For example, suppose there is only one valuable and non-verifiable task $B$. Then the principal and the agent can sign a long-term explicit contract $\Phi=\left\{a, p_{A}, F^{\alpha}, F^{P}\right\}$ on some verifiable task $A$ such that $v_{A}(a)=0$ for any level of $A$ and $c_{A}(0)=0$, $c_{A}^{\prime}(a)>0, c_{A}^{\prime \prime}(a)>0$. The transfer in this 'indirect' relational contract is then $p_{A}$, which compensates the agent for his provision of costly $b>0$. The principal and the agent can then also implicitly agree on a relational contract prescribing $a=0$ and $b>0$ on the equilibrium path, sustained by the threat of enforcing the explicit contract in case a defection is observed. Thus, the principal's grim trigger strategy is: if the agent deviates in the current period, the principal will implement the contract and demand task intensity $a$ on task $A$ forever after, imposing fines $F^{\alpha}$ whenever that task is not delivered. The agent's grim strategy is: if the principal deviates in the current period, the agent will not exert $b$ ever again in the future. In the punishment phase, the explicit contract is enforced: the principal will (have to) pay $p_{A}$ to the agent or incur fine $F^{P}$ and the agent will exert $a$ or incur fine $F^{\alpha}{ }^{8}$

In this case the participation constraints of the principal and the agent are respectively given by

$$
I R^{B C}-P: \frac{v_{B}(b)-p_{A}}{1-\delta} \geq 0
$$

and

$$
I R^{B C}-\alpha: \frac{p_{A}-c_{B}(b)}{1-\delta} \geq 0
$$

Note that under the explicit contract payment is non-discretionary. Then the principal can only profitably defect from the relational contract by levying the fine $F^{\alpha}$ on the agent at the end of the stage game for not having delivered the explicitly contracted intensity $a .^{9}$ After such a defection, the principal will have to pay $p_{A}$ forever after (optimal if $F^{P} \geq p_{A}$ ), in exchange for the valueless $a$, or otherwise pay the fine $F^{P}$ (optimal if $F^{P}<p_{A}$ ). Therefore, the relational incentive constraints of the principal is

$$
R I C^{B C}-P: \frac{v_{B}(b)-p_{A}}{1-\delta} \geq v_{B}(b)-p_{A}+F^{\alpha}-\frac{\delta \min \left[p_{A}, F^{P}\right]}{1-\delta} .
$$

Conversely, the agent can profitably defect from the relational contract by not providing $b$, but then he will have to simultaneously provide $a$ (optimal if $F^{\alpha} \geq c_{A}(a)$ ) or pay

\footnotetext{
${ }^{8}$ Note that since $p_{A}$ must be paid regardless of whether the agent exerts effort $b$, the distinction we made about simoultaneous and sequential ceases to be relevant under $B C$.

${ }^{9}$ Deviating by not paying the transfer $p_{A}\left(a_{E}\right)$ at the beiginning is not profitable, as then $P$ will not deliver $b_{I}$ and will also exercise the penalty $F_{G}$.
} 
the fine $F^{\alpha}$ (optimal if $F^{\alpha}<c_{A}(a)$ ). Therefore, the relational incentive constraints of the agent is

$$
R I C^{B C}-\alpha: \frac{p_{A}-c_{B}(b)}{1-\delta} \geq p_{A}-\min \left[F^{\alpha}, c_{A}(a)\right]+\delta \frac{p_{A}-\min \left[F^{\alpha}, c_{A}(a)\right]}{1-\delta} .
$$

We then obtain the following Lemma:

Lemma 4 (Optimality of Low Fines). The set of sustainable relational contracts with explicit overcontracting is non-empty and it is maximized by setting the fines at the minimum level necessary to induce compliance with the explicit contract, i.e. $F^{\alpha}=$ $c_{A}(a)$ and $F^{P}=p_{A}$.

Proof. Summing up $\left(\mathrm{RIC}^{\mathrm{BC}}-\mathrm{P}\right)$ in $(8)$ and $\left(\mathrm{RIC}^{\mathrm{BC}}-\mathrm{P}\right)$ in $(9)$, we obtain

$$
R I C^{B C}: \frac{v_{B}(b)-c_{B}(b)}{1-\delta} \geq v_{B}(b)+F^{\alpha}-\frac{\delta \min \left[p_{A}, F^{P}\right]}{1-\delta}-\frac{\min \left[F^{\alpha}, c_{A}(a)\right]}{1-\delta}+\delta \frac{p_{A}}{1-\delta} .
$$

By inspection, $F^{P}$ should be maximized, as it appears with a negative sign on the RHS of RIC; thus $F^{P}=p_{A}$. Now suppose $F^{\alpha}<c_{A}(a)$, then RIC becomes

$$
\frac{v_{B}(b)-c_{B}(b)}{1-\delta} \geq v_{B}(b)-\frac{\delta F^{\alpha}}{1-\delta}
$$

and as $F^{\alpha}$ appears with a negative sign on the right hand side of RIC, the set of sustainable constraints increases by raising $F^{\alpha}$ up to $c_{A}(a)$.

Lemma 4 provides a novel rationale for fines to be bounded above. The value of fines is to induce the parties to comply with the explicit contract in the punishment phase. There is no need for the fines to penalize the parties by imposing a cost that goes beyond the cost of compliance because non-compliance is perfectly detectable and there is nothing that fines can do which could not be done within the contract. Setting the fines higher than the cost of compliance for a party would reduce the feasibility of relational contracting by giving incentives to the other party to defect from the relational contract to cash the fine.

In the light of Lemma 4, the two relational constraints reduce to

$$
R \tilde{I} C^{B C}-P: \frac{\delta}{1-\delta} v_{B}(b) \geq c_{A}(a)
$$

and

$$
R \tilde{I} C^{B C}-\alpha: c_{A}(a) \geq c_{B}(b) .
$$

Consider (RĨC-P). By cooperating, the principal gains the surplus $v_{B}(b)$ from the valuable task in all future periods and gives up the gain from levying the fine $F^{\alpha}=c_{A}(a)$ 
in the current period. Consider ( $\mathrm{RI} C-\alpha)$. By cooperating, the agent incurs the cost $c_{B}(b)$ of undertaking the non-verifiable task $B$ whilst it saves on the cost $c_{A}(a)$ of undertaking the explicitely-contracted task $A$, in the current and in future periods. The relational constraints are independent of $p_{A}$ since $p_{A}$ must be paid regardless of whether $b$ is performed.

The following Proposition is then obtained.

Proposition 1 (The Value of Overcontracting Under BC). With the principal subject to BC, explicit (over-)contracting on a valueless but verifiable task $A$ acts as a threat that allows to sustain otherwise unfeasible relational contracts, implementing positive levels of the valuable but non-verifiable task B. The set of implementable task intensity satisfies

$$
\Phi^{B C}(b, \delta) \equiv \delta v_{B}(b)-c_{B}(b)(1-\delta) \geq 0 .
$$

with the highest implementable $b, b^{B C}(\delta)$, implemented by choosing intensity of task $A$ such that $c_{A}\left(a^{B C}\right)=c_{B}\left(b^{B C}\right)$.

Proof. The set of feasible relational contracts with overcontracting maximizes $b$ subject to $\left(\mathrm{IR}^{\mathrm{BC}}-\mathrm{P}\right),\left(\mathrm{IR}^{\mathrm{BC}}-\alpha\right),\left(\mathrm{RIC}^{\mathrm{BC}}-\alpha\right)$ and $\left(\mathrm{RIC}^{\mathrm{BC}}-\mathrm{P}\right)$. Since $p_{A}$ only affects $\left(\mathrm{IR}^{\mathrm{BC}}\right.$ $\mathrm{P}),\left(\mathrm{IR}^{\mathrm{BC}}-\alpha\right)$, any $p_{A} \in\left[c_{B}(b), v_{B}(b)\right]$ is feasible. Now let $b^{B C}(\delta)$ denote the highest sustainable $b$. We show that at $b^{O V}$, both $\left(\mathrm{RIC}^{\mathrm{BC}}-\alpha\right)$ and $\left(\mathrm{RIC}^{\mathrm{BC}}-\mathrm{P}\right)$ must be binding. Suppose by contradiction that $\left(\mathrm{RIC}^{\mathrm{BC}}-\mathrm{P}\right)$ is binding whilst $\left(\mathrm{RIC}^{\mathrm{BC}}-\alpha\right)$ is slack. Then $c_{A}(a)$ can be reduced so as to keep $\left(\mathrm{RIC}^{\mathrm{BC}}-\alpha\right)$ satisfied whilst loosening ( $\left.\tilde{\mathrm{IIC}}^{\mathrm{BC}}-\mathrm{P}\right)$, making higher levels of $b$ implementable: a contradiction. Suppose next that $\left(\mathrm{RIC}^{\mathrm{BC}}{ }_{-}\right.$ $\alpha)$ is binding whilst $\left(\mathrm{RIC}^{\mathrm{BC}}-\alpha\right)$ is slack. Then, we can increase $b$ and $a$ so as to leave $\left(\mathrm{RIC}^{\mathrm{BC}}-\alpha\right)$ binding and keep $\left(\mathrm{RIC}^{\mathrm{BC}}-\mathrm{P}\right)$ satisfied. Setting both $\left(\mathrm{RIC}^{\mathrm{BC}}-\alpha\right)$ and $\left(\mathrm{RIC}^{\mathrm{BC}}-P\right)$ binding we obtain expression (12).

Proposition 1 provides a rationale as to why explicit contracts can be signed but not applied, particularly when the principals in these contracts are members of a large hierarchical organizations, like Civil Servants procuring for a Government or Procurement Managers of large firms. Overcontracting can be a means to sustain relational contracting when discretionary transfers are not allowed. With overcontracting on tasks that are verifiable, costly to the agent but of no use for the principal, the principal can exchange discretionally the non-implementation of such explicit contract against the provision of non contractible tasks that are costly to the agent and valuable for the principal. Of course, all this relies on our choice to focus on 'benevolent' employees or civil servants. As mentioned in introduction, the same strategies could be used by non-benevolent employees to enforce implicit corrupt deals trading contract non-enforcements against bribes or private benefits $\mathrm{B}$.

We have so far assumed that the verifiable task $A$, contracted upon to induce effort on task $B$, is valueless to the principal. The Proposition below shows however that 
when explicit contracts are only used as threats, it is indeed optimal to write the contract on tasks that are valueless to the principal as this helps to enlarge the set of sustainable relational contracts under BC.

Proposition 2 With the principal subject to BC, other things equal, the set of sustainable task intensity that is achievable through overcontracting on a verifiable task $A$ is maximized when $A$ is valueless to the principal.

Proof. Let $v_{A}(a)$ denote the value of the verifiable task $A$. The relational contract of the principal modifies to

$$
R I C^{B C}-P: \frac{v_{B}(b)-p_{A}}{1-\delta} \geq v_{B}(b)-p_{A}+F^{\alpha}+\frac{v_{A}(a)-\delta \min \left[p_{A}, F^{P}\right]}{1-\delta} .
$$

whilst $v_{A}(a)$ has no effect on $\left(\mathrm{RIC}^{\mathrm{BC}}-\mathrm{P}\right),\left(\mathrm{IR}^{\mathrm{BC}}-\mathrm{P}\right)$ and $\left(\mathrm{IR}^{\mathrm{BC}}-\alpha\right)$. Since the LHS of $\left(\mathrm{RIC}^{\mathrm{BC}}-\mathrm{P}\right)$ is independent of $v_{A}(a)$ whilst the RHS is increasing in $v_{A}(a)$ and since the set of sustainable relational contracts is maximized when $\left(\mathrm{RIC}^{\mathrm{BC}}-\mathrm{P}\right)$ binds, the result follows.

Intuitively, if task $A$ were valuable to the principal, the principal would have more to gain from defaulting by enforcing the explicit contract. His payoff in the punishment phase would therefore increase, thus reducing the gain from sticking to the relational contract.

\section{The General Value of Contracts as Threats}

The results in the previous section show that explicit contracting on a non-valuable task can make relational contracts sustainable when discretionary transfers are not allowed. In fact we now show that this overcontracting might facilitate relational contracts on valuable but non-contractible tasks even in the absence of bureaucratic control.

Assuming away BC and maintaining for the moment the assumption that renegotiation is prohibitively costly, consider the optimal relational contract when both discretionary transfers and overcontracting are allowed (BC). Without loss of generality, with regard to the timing of the discretionary payment $t_{B}$ we focus on the case of simultaneous timing (Stage game 1).

Keeping the same notation as in the previous section, with discretionary transfers and overcontracting the participation constraints become

$$
\begin{array}{cl}
I R^{O V}-P & : \quad \frac{v_{B}(b)-p_{A}-t_{B}}{1-\delta} \geq 0 \\
I R^{O V}-\alpha & : \quad \frac{t_{B}+p_{A}-c_{B}(b)}{1-\delta} \geq 0
\end{array}
$$


Suppose now that $t_{B} \geq 0$ : the discretionary transfer takes the form of a payment from the principal to the agent. A deviation by the principal then consists in not paying $t_{B}$ when $b$ was observed and in levying the fine $F^{\alpha}$ on the agent for not exerting $a$. A deviation by the agent consists in exerting $a$ (or incurring the fine) instead of exerting $b$. The relational incentive constraints are therefore

$$
R I C^{O V}-P: \frac{v_{B}(b)-p_{A}-t_{B}}{1-\delta} \geq v_{B}(b)-p_{A}+F^{\alpha}-\frac{\delta \min \left[p_{A}, F^{P}\right]}{1-\delta}
$$

and

$$
R I C^{O V}-\alpha: \frac{t_{B}+p_{A}-c_{B}(b)}{1-\delta} \geq t_{B}+p_{A}-\min \left[F^{\alpha}, c_{A}(a)\right]+\delta \frac{p_{A}-\min \left[F^{\alpha}, c_{A}(a)\right]}{1-\delta} .
$$

Summing up the two constraints, substituting for the optimal fines (Lemma 4 still applies) and simplifying, we obtain

$$
R I C^{O V}: \delta v_{B}(b)-c_{B}(b)-(1-\delta) t_{B}+\delta c_{A}(a) \geq 0
$$

Since LHS of RIC decreases with $t_{B}$ and it is unaffected by $p_{A}$, the set of sustainable relational contracts is maximized at $t_{B}=0$ with $p_{A}$ chosen so as to satisfy $\mathrm{IR}^{\mathrm{OV}} \mathrm{S}$. Compared to $\left(\mathrm{RIC}^{\mathrm{ST}}\right)$ under standard relational contracting (expression 6), there is an additional positive term, $\delta c_{A}(a)$, which is the cost of deviation for the agent given by the cost of complying with the explicit contract. This leads us to the following Lemma.

Lemma 5 (Sub-optimality of Discretionary Transfers). With overcontracting, discretionary transfers are suboptimal: in any relational efficient equilibrium $t_{B}=0$.

Proof. Suppose that $t_{B} \leq 0$. In this case a deviation by the principal only results in the levying of the fine; since $t_{B}$ is non-positive, it is the agent who can withhold payment to the principal and not vice versa. The relational incentive constraints therefore become

$$
R \tilde{I} C^{O V}-P: \frac{v_{B}(b)-p_{A}-t_{B}}{1-\delta} \geq v_{B}(b)-t_{B}-p_{A}+F^{\alpha}-\frac{\delta \min \left[p_{A}, F^{P}\right]}{1-\delta}
$$

and

$$
R \tilde{I} C^{O V}-\alpha: \frac{t_{B}+p_{A}-c_{B}(b)}{1-\delta} \geq p_{A}-\min \left[F^{\alpha}, c_{A}(a)\right]+\delta \frac{p_{A}-\min \left[F^{\alpha}, c_{A}(a)\right]}{1-\delta} .
$$

Summing up these two constraints, we have

$$
R I C^{O V}: \delta v_{B}(b)+(1-\delta) t_{B}-c_{B}(b)+\delta c_{A}(a) \geq 0
$$

and the set of sustainable relational contracts is maximized at $t_{B}=0$.

Before giving the intuition for the above Lemma, we state the following Proposition. 
Proposition 3 (Rewarding A to Obtain B with no BC) (i) Explicit (over-)contracting on a valueless but verifiable task allows to sustain higher levels of the valuable and non-verifiable task than standard relational contracting under either sequential or simultaneous timing. The set of sustainable relational contracting is given by

$$
\Phi^{O V}(b, \delta) \equiv \Phi^{B C}(b) \equiv \delta v_{B}(b)-c_{B}(b)(1-\delta) \geq 0,
$$

with the highest implementable $b, b^{O V}(\delta)$, obtained by choosing intensity of task $A$ such that $c_{A}\left(a^{O V}\right)=c_{B}\left(b^{O V}\right)$ and solving $\Phi^{O V}\left(b^{O V}, \delta\right)=0$. (ii) The set of feasible relational contracting under overcontracting is strictly greater than under standard relational contracting:

$$
\Phi^{O V}(b, \delta)>\Phi_{S e q}^{S T}(b, \delta)>\Phi_{S m l}^{S T}(b, \delta) .
$$

Proof. Substitute for $t_{B}=0$ in (15) and note that expression (15) is increasing in $c_{A}(a)$. Since $\left(\mathrm{RIC}^{\mathrm{OV}}-\alpha\right)$ in expression (14) reduces to $c_{A}(a) \geq c_{B}(b)$ and since (11) is independent of $c_{A}(a)$, it follows that the set of feasible contracts is maximized at $c_{A}(a)=c_{B}(b)$. This gives $\Phi^{O V}(b, \delta)$.

Compared to standard relational contracting, overcontracting generates two effects. First, it introduces the agent's obligation to comply with the explicit contract (or pay the fine) and it gives the principal the ability to levy the fine after observing whether the agent complied with the implicit contract. By allowing the principal to react immediately to a deviation by the agent, overcontracing generates a sequentiality that weakly reduces the agent's gains from defection (strictly when the stage game is simultaneous). Through an appropriate choice of the cost of compliance, this effect can completely remove the incentives of the agent to default. In particular, by choosing intensity of task $A$ such that $c_{A}\left(a^{O V}\right)=c_{B}\left(b^{O V}\right)$, the agent is made indifferent between complying with the implicit contract and exert $b^{O V}$ and defaulting and exert $a^{O V}$ (or pay the fine $F^{\alpha}=c_{A}\left(a^{O V}\right)$ ). Other things equal, overcontracting can therefore do at least as well as standard relational contracting with sequential timing where the agent moves first and only (RIC-P) matters.

Second, overcontracting affects payoffs in the punishment phase starting in the period after a defection takes place. By requiring parties to comply with a wasteful explicit contract, overcontracting strengthens the punishment phase. This effect explains why overcontracting can do also strictly better than standard relational contracting with sequential timing.

By creating - through sequentiality - a cost of defaulting for the agent, and by strengthening the punishment phase, the use of explicit contracts as threats is more efficient for sustaining relational contracting than the use of discretionary transfers. The positive non-pecuniary effect of overcontracting as an 'inefficient threat' (the second effect described above), is related to the positive effect of 'money burning' in Clark-Grooves-Vikery mechanisms and relational contracting models with subjective 
evaluation, like McLeod (2003), Levine (2003), and Fuchs (2007); and it is reminding of the positive effect of inefficient ownership structures on investment in long term relationships with 'hold up' problems, stressed by Halonen (2002) with respect to joint ownership and generalized by Blonski and Spagnolo (2004).

\section{Renegotiation and Short Term Contracts}

In the basic model we have assumed that the parties can commit to long-term explicit contracts. We now consider the possibility that explicit contracts can be renegotiated at low cost later than period 0 and in particular after a defection from a relational contract is observed. Clearly, this possibility has no effect under standard relational contracting as no explicit contracting is present. Consider therefore the case of overcontracting on $a$ to sustain otherwise unfeasible levels of $b$. We will discuss both long and short-term contracts.

In the long-term contracting case studied in the previous sections, if renegotiation costs are sufficiently small, after an implicit contract breaks up because of a defection, it is efficient for the principal and the agent to renegotiate the explicit contract to $a=0$, given that task $a$ is costly but valueless. Let $z>0$ denote the cost of renegotiating the explicit contract. Then in the renegotiation the principal and the agent will bargain to share the gain from not implementing the inefficient explicit contract net of the renegotiation cost: $\frac{\delta c_{A}(a)}{1-\delta}-z$. When $z \geq \frac{\delta c_{A}(a)}{1-\delta}$ at $c_{A}(a)=c_{B}(b)$, we are in the situation of Section 4 , so we focus here on $z<\frac{\delta c_{B}\left(b^{O V}\right)}{1-\delta}$.

Assuming $0 \leq z<\frac{\delta c_{A}}{1-\delta}$ and Nash bargaining in the renegotiation phase, the agent obtains

$$
\frac{\delta\left(p_{A}-c_{A}\right)}{1-\delta}+\frac{1}{2}\left(\frac{\delta c_{A}}{1-\delta}-z\right)=\frac{\delta\left(p_{A}-\frac{1}{2} c_{A}\right)}{1-\delta}-\frac{z}{2}
$$

whilst the principal obtains

$$
\frac{-\delta p_{A}}{1-\delta}+\frac{1}{2}\left(\frac{\delta c_{A}}{1-\delta}-z\right)=-\frac{\delta\left(p_{A}-\frac{1}{2} c_{A}\right)}{1-\delta}-\frac{z}{2} .
$$

Therefore, the relational incentive constraints of the principal and the agent become respectively (fines are unchanged)

$$
\begin{gathered}
R \hat{I} C^{O V}-P: \frac{v_{B}(b)-p_{A}}{1-\delta} \geq v_{B}(b)-p_{A}+c_{A}(a)-\delta \frac{p_{A}-\frac{1}{2} c_{A}(a)}{1-\delta}-\frac{z}{2} \\
R \hat{I} C^{O V}-\alpha: \frac{p_{A}-c_{B}(b)}{1-\delta} \geq p_{A}-c_{A}(a)+\delta \frac{p_{A}-\frac{1}{2} c_{A}(a)}{1-\delta}-\frac{z}{2}
\end{gathered}
$$

We then obtain the following Lemma. 
Lemma 6 With overcontracting and renegotiation cost of $z$, the set of sustainable relational contracts is given by

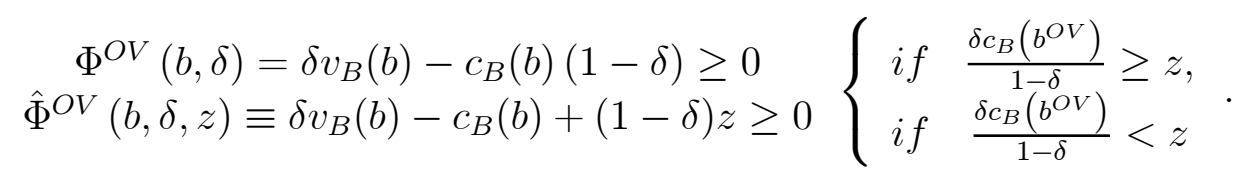

where $\tilde{\Phi}^{O V}(b, \delta, z)$ is increasing in $z$ with

$$
\tilde{\Phi}^{O V}(b, \delta, z v) \leq \Phi^{O V}(b) .
$$

The level of overcontracting, $c_{A}(b)-c_{B}(b)$, is non-increases in $z$.

Proof. Suppose that $\frac{\delta c_{B}\left(\hat{b}^{O V}\right)}{1-\delta} \geq z$. The two relational constraints simplify to

$$
R \hat{I} C^{O V}-P: \delta v_{B}(b) \geq c_{A}(a)\left(1-\frac{\delta}{2}\right)-(1-\delta) \frac{z}{2}
$$

and

$$
R \hat{I} C^{O V}-\alpha: c_{A}(a)\left(1-\frac{\delta}{2}\right) \geq c_{B}(b)-(1-\delta) \frac{z}{2}
$$

Following the same reasoning as in Proposition 1, the set of sustainable relational contracts is found by choosing $a$ and $b$ such that $\left(\mathrm{RIC}^{O V}-\alpha\right)$ and $\left(\mathrm{RiC}^{O V}-\mathrm{P}\right)$ are binding. This gives $a$ and $b$ solving

$$
\begin{gathered}
c_{A}\left(\hat{a}^{O V}\right)\left(1-\frac{\delta}{2}\right)=c_{B}\left(\hat{b}^{O V}\right)-(1-\delta) \frac{z}{2}, \\
\delta v_{B}\left(\hat{b}^{O V}\right)-c_{B}\left(\hat{b}^{O V}\right)+(1-\delta) z=0 .
\end{gathered}
$$

with $\frac{\delta}{1-\delta} c_{A}\left(\hat{a}^{O V}\right)-z=2 \frac{\delta c_{B}\left(b^{O V}\right)-z(1-\delta)}{(1-\delta)(2-\delta)}$ which is positive for $\frac{\delta c_{B}\left(\hat{b}^{O V}\right)}{1-\delta} \geq z$. Thus the above is a solution for $\frac{\delta c_{B}\left(\hat{b}^{O V}\right)}{1-\delta} \geq z$. Suppose now that $\frac{\delta c_{B}\left(\hat{b}^{O V}\right)}{1-\delta}<z$. Then the parties will prefer not to renegotiate the contract during the punishment phase and the analysis is equivalent to the case developed in Section 4. Thus the set of feasible relational contracts is then given by (16).

By increasing the payoffs of the parties in the punishment phase, renegotiation reduces the sustainability of relational contracts with overcontracting. As payoffs raise more the lower is the renegotiation cost, the set $\hat{\Phi}^{O V}(b, \delta, z)$ of sustainable relational contracts shrinks as $z$ decreases.

As for other inefficient contractual devices delivering strategic advantages, like strategic delegation contracts, the possibility of renegotiating at low costs tends to 
undermine the benefit of the arrangement (see e.g. Dewatripont 1988, and Katz 2001). For example, in Halonen (2002) the positive effect of joint ownership is the smaller the lower are renegotiation costs, and the effect vanishes all together when the renegotiation cost is zero. Similarly, Kocksen and Ok (2004) found recently additional strategic effects of contractual delegation, but only with costly renegotiation. These effects vanish when renegotiation becomes costless.

However, in our setup, even in the degenerate case of $z=0$, renegotiation does not completely eliminate the value of overcontracting. This is due to the first effect of overcontracting described above, namely the ability of overcontracting to create sequentiality when the original stage game is simultaneous. Indeed, by inspection, at $z=0, \tilde{\Phi}^{O V}(z=0, b)=\Phi_{\text {Seq }}^{S T}(z=0, b)$ in $(7)$. The following Proposition is then obtained.

Proposition 4 (Overcontracting with Renegotiation). Renegotiation reduces the set of sustainable relational contracts sustainable with overcontracting, the more the lower the renegotiation cost $z$. However: (i) As long as $z \neq 0$, overcontracting strictly dominates standard relational contracting, as $\Phi_{\text {Ov }}^{z}(b)>\Phi_{\text {Seq }}(b)>\Phi_{\text {Sml }}(b)$. (ii) At $z=0$, overcontracting weakly dominates standard relational contracting, and strictly dominates it when the timing of the stage game is simultaneous or if there is $B C$.

Proposition 4 stems from a combination of two factors. First, when renegotiation occurs, the agent bears a cost of defaulting which is given by the cost of complying with the explicit contract at the time of defaulting. Second, such cost is endogenous: when renegotiation becomes less costly, the parties can design a more demanding explicit contract so as to leave the payoff of the agent unchanged. Thus, regardless of the cost of renegotiation, the agent can be given incentives not to default.

In our simple framework we also obtain this result when only one-period explicit contracts are available.

Corollary 1 The set of sustainable relational contracts with overcontracting when only short term contracts are possible is the same as that with long-term overcontracting and costless renegotiation $(z=0)$. Hence, also when only short term contracts exist overcontracting weakly dominates standard relational contracting, and strictly dominates it when the timing of the stage game must be simultaneous or if there is $B C$.

Proof. Suppose now that the principal and the agent agree to a short term contract and to renegotiate it as long as parties do not defect. The relational incentive constraints of the principal and the agent become respectively (fines are unchanged)

$$
R I C_{\text {Short }}^{O V}-P: \frac{v_{B}(b)-p_{A}}{1-\delta} \geq v_{B}(b)-p_{A}+c_{A},
$$


and

$$
R I C_{\text {Short }}^{O V}-\alpha: \frac{p_{A}-c_{B}(b)}{1-\delta} \geq p_{A}-c_{A}(a)
$$

Simplifying

$$
R I C_{\text {Short }}^{O V}-P: \delta\left(v_{B}(b)-p_{A}\right)-c_{A}(1-\delta) \geq 0
$$

and

$$
R I C_{\text {Short }}^{O V}-\alpha: \delta p_{A}-c_{B}(b)+c_{A}(1-\delta) \geq 0 .
$$

The set of relational contracts is maximized by setting $c_{B}(b)=c_{A}=p_{A}$, which yields

$$
\Phi_{\text {Short }}^{O V}(b) \equiv \delta v_{B}(b)-c_{B}(b) \geq 0,
$$

which is equal to $\Phi^{O V}(b)$, the set of sustainable relational contracts with long-term contracting and renegotiation, given by (18). This proves the first statement. The second follows directly from the Proposition.

\section{Distributional Effects and Financing Constraints}

In this section we make the extreme assumption that renegotiation is costless (or that only one-period explicit contracts can be signed), and show that even with sequential timing there might still be reasons for using overcontracting to sustain relational contracts.

Distributional effects. Overcontracting has important distributional consequences. In standard relational contracting, an agent who gets no surplus gets no long-term benefit from continuing the contract, and will therefore default if the timing of the exchange cannot be properly adjusted. Indeed, without overcontracting the condition $t_{B} \geq c_{B}(b) / \delta$ is necessary for $\left(\mathrm{RIC}^{\mathrm{ST}}-\alpha\right)$ to be satisfied, if for any reason the timing of the exchange must follow Stage Game 1 or 2. Thus, under standard relational contracting, the agent must be given a rent of at least $(1-\delta) c_{B} / \delta$. Under overcontracting, instead, an agent who gets no surplus can still get a long-term benefit from continuing the contract because there is a cost of defaulting. In the case of no renegotiation, for example, when the agent defaults he triggers the implementation of the explicit contract, which costs him $c_{A}(a)$ (or the correspondent fine $F^{\alpha}$ ). Since defaulting saves the agent the amount $c_{B}(b)$, choosing $a$ such that $c_{A}(a)=c_{B}(b)$ suffices to remove any incentive to default. We then obtain:

Corollary 2 Even if $z=0$ or only short term contracts are available, if the timing of the exchange follows STAGE GAme 1 or 2 overcontracting on A allows the principal to implement any level of $B$ that is feasible under standard relational contracting whilst retaining a greater share of the surplus. With overcontracting, no rent is needed to discipline the agent. 
Proof. (i) Consider the case of overcontracting with no renegotiation. We know from the Proof of Proposition 1 that the set of sustainable relational contracts is maximized by choosing $a$ such that $c_{A}(a)=c_{B}(b)$. The result then follows by noting that $\left(\mathrm{RIC}^{\mathrm{BC}}-\alpha\right)$ in expression (9) is satisfied at $p_{A}(b)=c_{A}(a)=c_{B}(b)$ and fines satisfying Lemma 4. (ii) Now consider the case of renegotiation. We know from Lemma 6 that the set of sustainable relational contracts is maximized by choosing $a$ such that $c_{A}(a)\left(1-\frac{\delta}{2}\right)=c_{B}(b)-(1-\delta) \frac{z}{2}$. The result then follows by noting that $\left(\mathrm{RIC}^{\mathrm{OV}}-\alpha\right)$ in expression $(17)$ is satisfied at $p_{A}(b)=c_{B}(b)$ and $a$ such that $c_{A}(a)\left(1-\frac{\delta}{2}\right)=c_{B}(b)-(1-\delta) \frac{z}{2}$.

Financing constraints. This same ability of overcontracting to solve problems

linked to the sequence of exchange has also important effects when there are financial constraints that prevent agents to implement the efficient sequence of the exchange.

Suppose, for example, that the principal has all bargaining power and therefore is the residual claimant of the future rent from the relationship. Suppose further that the task is a nonverifiable but costly, and that the agent does not have own money to finance such an investment and is financially constrained (cannot borrow it). Since the principal has the bargaining power, we know that it would be efficient that he requires the agent to invest first, according to Stage Game 3. However, if the agent is financially constrained, it will not be able to invest first and an inefficient timing will have to be chosen that will also cost the principal part of the obtainable rent, as shown by the Corollary above.

Overcontracting on a useless but verifiable task, even if $z=0$ or only short term contracts are available, allows to solve the problem thanks to its distributional effects. It allows the principal to pay first $p_{A}$ to the agent, which allows the agent to finance the agreed upon investment $b$, without inducing any inefficiency and rent redistribution. We thus have:

Corollary 3 Even if $z=0$ or only short term contracts are available, overcontracting allows to sustain an efficient relational contracts when financing constraints would not allow it otherwise.

\section{Conclusions}

We have shown that explicit contracts can not only be seen as safe "boundaries" within which relational contracts operate better, as suggested by Klein (2000), or as constraints on discretion that hinder relational contracting, as suggested by Bernheim and Whinston (1998), but also as credible "threats" that - not being applied - actively help governing relationships. When this is the case, overcontracting may be observed, as explicit and not applied contracts on verifiable tasks with little value offer discretionary 
actions (legal penalties) may enforce better relationships than standard relational contracts. This result could explain some cases in which we observe that explicit contracts regulate and reward apparently useless tasks and are not actually applied, and can be seen as the complement to the undercontracting result in Bernheim and Whinston (1998), where explicit contracting on valuable tasks is reduced to facilitate relational contracts. Both these results are based on externalities between contractible and noncontractible tasks, and therefore open the way to many new questions on optimal job and organizational design.

Also, as mentioned earlier, we have been mainly focussing on legal and productive noncontractible tasks B (or effort, or investments). However, illegal tasks, bribes and private benefits also cannot be explicitly contracted (since they are forbidden, they cannot be brought in front of a court), so that overcontracting may help to sustain corrupt relational exchanges exactly as it helps productive ones. The trade off between the positive and negative effects of overcontracting, and the optimal regulatory response to it in different legal and cultural environments appears therefore and important question to address in future work. 


\section{References}

[1] Abreu, D., (1988), "On the Theory of Infinitely Repeated Games with Discounting", Econometrica 56, 383-396.

[2] Albano, Dini, Spagnolo, (2008), "Strumenti a Sostegno della Qualità negli Acquisti Pubblici," Quaderno Consip I//2008.

[3] Banfield, E., (1975), "Corruption as a Feature of Governmental Organization", Journal of Law and Economics, 1975, 18, 587-605.

[4] Baker, G., Gibbons, R. and Murphy, K.J., (1994), "Subjective Performance Measures in Optimal Incentive Contracts", Quarterly Journal of Economics, 109, 112556.

[5] Baker, G., Gibbons, R. and Murphy, K.J., (2001), "Relational Contracts and the Theory of the Firm", Quarterly Journal of Economics, 117(1), 39-84.

[6] Baker, G., Gibbons, R. and Murphy, K.J., (2006), "Contracting for Control", unpublished manuscript, HBS, MIT and USC.

[7] Bernheim, D. and Whinston, M., (1998), "Incomplete Contracts and Strategic Ambiguity," American Economic Review, 88, 902-32.

[8] Becker, G., (1968), "Crime and Punishment: An Economic Approach," Journal of Political Economy 76(2), 169-217.

[9] Blonski, M. and Spagnolo, G., (2003), "Relational Contracts and Property Rights," CEPR Discussion Paper No. 3460.

[10] Bragelien, I., (2003), "Asset Owenership and Relational Contracts," unpublished manuscript, Norwegian School of Economics and Business Administration.

[11] Bull, C. (1987), "The Existence of Self-Enforcing Implicit Contracts", Quarterly Journal of Economics, 102(1), 147-159.

[12] Calzolari, G., and Spagnolo, G., (2007), "Relational Procurement," manuscript, Universities of Bologna and of Rome 'Tor Vergata'.

[13] Dewatripont, M. (1988), "Commitment Through Renegotiation-Proof Contracts with Third Parties," Review of Economic Studies, 55, 377-90.

[14] Fehr, E., Brown, M. and Falk, A. (2004), "Relational Contracts and the Nature of Market Interactions", Econometrica, 72(3), 747-780. 
[15] Fuchs, W. (2007), "Contracting with Repeated Moral Hazard and Private Evalutions," American Economic Review, 97(4), 1432-1448.

[16] Garvey, G., (1995), "Why Reputation Favors Joint Ventures over Vertical and Horizontal Integration: A Simple Model," Journal of Economic Behavior and Organization, 28, 387-97

[17] Halonen, M., (2002), "Reputation and The Allocation of Ownership", The Economic Journal, 112, 539-558.

[18] Holmstrom, B. and Milgrom, P. (1991), "Multi-task Principal-Agent Analysis: Incentive Contracts, Asset Ownership and Job Design," Journal of Law, Economics and Organization, 7, 24-52.

[19] Katz, M. (1991), "Game-Playing Agents: Unobservable Contracts as Precommitments," RAND Journal of Economics, 22(3), 307-28.

[20] Kelman, S. (1990), Procurement and Public Management: The Fear of Discretion and the Quality of Goverment Performance AEI Press.

[21] Kerr, S. (1975), "On the Folly of Rewarding A, While Hoping for B," Academy of Management Journal, 18(4), 769-783.

[22] Klein, B. (2000), "The Role of Incomplete Contracts in Self-Enforcing Relationships," Revue D'Economie Industrielle, 92 67-80

[23] Klein, B. and Leffler, K., (1981), "The Role of Market Forces in Assuring Contractual Performance", Journal of Political Economy, 89, 615-41.

[24] Kockesen, L. and Ok, E., (2004), "Strategic Delegation By Unobservable Incentive Contracts," Review of Economic Studies, 71(2), 397-424.

[25] Levin, J., (2003), "Relational Incentive Contracts", American Economic Review, $93(3), 835-847$.

[26] Macaulay, S., (1963), "Non Contractual Relations in Business: A Preliminary Study", American Sociological Review, 28, 55-67.

[27] MacLeod, W.B. and Malcomson, J.M., (1989), "Implicit Contracts, Incentive Compatibility, and the Involuntary Unemployment", Econometrica, 57(2), 447480 .

[28] MacLeod, W. B. and Malcomson, J.M., (1998), "Motivation and Markets", American Economic Review, 88(3), 388-411. 
[29] MacLeod, W.B., (2003), "Optimal Contracting with Subjective Evaluation," American Economic Review, 93(1), 216-240.

[30] MacLeod, W.B., (2007), "Reputations, Relationships and Contract Enforcement," Journal of Economic Literature, 45(3), 595-628.

[31] Pearce, D. and Stacchetti, E., (1998), "The Interaction of Implicit and Explicit Contracts in Repeated Agency", Games and Economic Behavior, 23(1), 75-96.

[32] Simon, H., (1951), "A Formal Theory of the Employment Relationship," Econometrica, 19, 293-305.

[33] Schmidt, K., and Schnitzer, M., (1995), "The Interaction of Explicit and Implicit Contracts," Economic Letters, 48, 193-199.

[34] Telser, L., (1980), "A Theory of Self-Enforcing Agreements," Journal of Business, $53(1), 27-44$. 